The March issue, Vol. 16, No. 3, page 379 inadvertently ran in authors' names, removing the spaces between the first and last names and deleted the "and" between the author's names. The printer sincerely regrets the error. The corrected author name line is shown below.

\title{
Upper Stability Island of the Quadrupole Mass Filter with Amplitude Modulation of the Applied Voltages
}

\author{
N. V. Konenkov, A. N. Korolkov, and Marat Machmudov \\ Ryazan State Pedagogical University, Ryazan, Russia
}

Modulation of the voltages applied to a quadrupole mass filter (QMF), either RF or RF and DC, leads to splitting of the stability region into islands of stability. The ion optical properties, such as transmission, resolving power and peak tails of the first upper stability islands have been investigated by numerical simulation of ion trajectories. The dependence of the location of this island on the amplitude of the modulation and the parameter $\nu=\omega / \Omega=\mathrm{Q} / \mathrm{P}$ where $\omega$ is modulation frequency, $\Omega$ is main angular radio frequency, and $Q$ and $P$ are integers, is calculated in detail. Different methods of adjusting the QMF resolution are examined. It is found that operation at the upper and lower tips of the stability islands created by amplitude modulation of the RF voltage is preferred, because of the technical simplicity of this method and a reduction of the required separation time. Amplitude modulation improves the performance of a QMF constructed with round rods, in comparison to perfect quadrupole fields. For example, with amplitude modulation of the $R F$, to reach a resolution of $R_{0.1}=1200$ requires only about $75 \mathrm{RF}$ cycles of ion motion in a quadrupole field created by round rods. (J Am Soc Mass Spectrom 2005, 16, 379-387) (C) 2005 American Society for Mass Spectrometry 